\title{
Vector competence of the tick Ixodes sinensis (Acari: Ixodidae) for Rickettsia monacensis
}

\author{
Xiaodong Ye ${ }^{1,2}$, Yi Sun ${ }^{1 *}$, Wendong $\mathrm{Ju}^{3}$, Xin Wang ${ }^{4}$, Wuchun Cao ${ }^{1}$ and Mingyu Wu ${ }^{1}$
}

\begin{abstract}
Background: Cases of Mediterranean Spotted Fever like rickettsioses, caused by Rickettsia monacensis, have become more common in the last 10 years. In China, natural infection of $R$. monacensis in various tick species has been confirmed but the vector(s) of $R$. monacensis have not been recorded.

Methods: The prevalence of $R$. monacensis in $>1500$ Ixodidae ticks from central and southern China was determined using centrifugation-shell vial culture and polymerase chain reaction techniques. The predominant species, Ixodes sinensis, harbored a natural infection of $R$. monacensis and was assumed to be a vector candidate of $R$. monacensis. Experimental transmissions were initialized by infecting Rickettsia-free tick colonies with $R$. monacensis using capillary tube feeding (CTF) or immersion techniques. Transstadial and transovarial transmissions, and transmission from ticks to mice, were conducted under laboratory conditions.
\end{abstract}

Results: $R$. monacensis was isolated and identified from hemolymph of Ixodes sinensis using molecular techniques. Transovarial transmission of $R$. monacensis from infected $O /$. sinensis to offspring was documented and infected offspring successfully passed Rickettsia to mice. Transstadial transmission rates were 58\% in larva to nymph and 56\% in nymph to adult stages. Infected nymphs and adults were also able to infect mice.

Conclusions: I. sinensis is a competence vector for $R$. monacensis as demonstrated by natural infection and transmission studies.

Keywords: Rickettsia monacensis, Ixodes sinensis, Vector competence

\section{Background}

Rickettsiae are obligate intracellular, gram-negative, alphaproteobacteria usually transmitted by arthropod vectors. They cause various human diseases including emerging spotted fever rickettsiosis [1]. Since the initial study of the spotted fever group (SFG) rickettsia by Ricketts (1906) more than 27 described species and uncharacterized strains have been associated with spotted fever rickettsiosis $[2,3] . R$. monacensis was first isolated and characterized in 2002 from Ixodes ricinus ticks collected in Munich, Germany [4]. Five years later, $R$. monacensis was identified from Mediterranean Spotted Fever (MSF)-like patients in Spain [5]. General discomfort, headache, and joint pain, a nonpruritic, disseminated maculopapular rash or an erythematous rash with no inoculation escharare typical

\footnotetext{
* Correspondence: sunyi73@gmail.com

'State Key Laboratory of Pathogen and Biosecurity, Beijing Institute of Microbiology \& Epidemiology, No. 20 Dongdajie Str. Fengtai District, Beijing, People Republic of China

Full list of author information is available at the end of the article
}

symptoms. Since 2007, MSF-like cases have been documented in Italy [6], Croatia [7] and the Republic of Korean (EU883092, FJ009429). To determine possible arthropod vectors, vertebrate reservoirs, and geographic ranges, epidemiological surveys for $R$. monacensis have been performed in several European countries [8-11]. The prevalence of $R$. monacensis in I. ricinus ranged from 4\% (Spain), 8.6\% (Germany), and 12.2\% (Slovakia) to $52.9 \%$ (Bulgaria) [12,13]. $R$. monacensis was also found in Ixodes persulcatus from mainland China [14], Ixodes nipponensis [15] and Haemaphysalis longicornis [16] from the Republic of Korea, and Haemaphysalis punctata [8] from Italy, suggesting that many tick species are involved in the zoonotic cycles and wide geographic range of $R$. monacensis. However, the presence of $R$. monacensis in these ticks does not prove that they are competent vectors of $R$. monacensis. Transmission data provides better evidence for the potential of Ixodid ticks to serve as vectors for $R$. monacensis. 
Tick-borne rickettsial diseases are a significant problem in China. Over the last 5 years, tick populations have generally increased and this has led to an increase in human tick bites [17]. Two phenomena are striking. First, thousands of hospitalized patients have unexplained febrile illnesses coinciding with the period of greatest tick activity. Data on clinical symptoms, history of exposure to ticks, and presumptive therapy strongly suggests that some of the patients are infected by SFG rickettsial pathogens [17]. Second, the range of human cases appears to be expanding southward. Many human cases with typical spotted fever symptoms have been found in central and southern China. While some common factors may be at play, the mechanisms behind infection and range expansion have not been fully clarified. Infections may be influenced by climate changes, potential vector ticks, host population dynamics, and human behaviour changes. Due to current diagnostic techniques that depend on clinical symptoms and serological and/or commercially available genus-specific PCR assays, detailed information about the pathogens and the vector ticks is scarce.

Knowledge of tick borne rickettsiosis ecology is essential to understand the potential threat of emerging Rickettsia spp. and vector ticks in central and southern China.To address this issue, we surveyed for Rickettsia spp. in Chinese Ixodid ticks. Our focus was on species which frequently bite tourists and residents and are prevalent throughout pastoral and forest areas from Henan, Hubei, Anhui, Shandong, Jiangsu and Zhejiang provinces. The survey was conducted during 2009-2013. R. monacensis was successfully isolated and identified from $I$. sinensis in Guangshan county, Henan province. I. sinensis is closely related to the well-known vector $I$. ricinus inmorphology, phylogeny and blood feeding behavior. I. sinensis was therefore assumed to be a probable vector of $R$. monacensis and its capability for transtadial and transovarial transmission was determined.

\section{Methods}

\section{Ticks}

Questing ticks were collected on vegetation using the blanket sweeping method in the survey sites from January, 2009 to January 2013 (Figure 1).After identification using standard taxonomic keys, hemolymph, for culture of $R$. monacensis, was collected from each tick from a cut leg using a sterile capillary tube [18]; the body of each tick was simultaneously screened for presence of the pathogen. To obtain a Rickettsia free colony, engorged females $I$. sinensis were maintained individually in $1.5 \mathrm{~cm}$ diameter test tubes for egg-laying under a $16 \mathrm{hr}$ light $/ 8 \mathrm{hr}$ dark photoperiod at $22^{\circ} \mathrm{C}$ within glass desiccators above a saturated aqueous solution of $\mathrm{K}_{2} \mathrm{SO}_{4}$ that maintained 97\% RH [19]. After hatching, about 50 filial larvae were sampled from each adult. Both sampled filial larvae and their mother were tested for Rickettsia spp. by PCR screening described as follows. Only the Rickettsianegative filial progeny from Rickettsia free mothers were used in the experimental colony. The Rickettsia free nymph and adult colonies were harvested from Rickettsia free larvae and nymph colonies fed on pathogen free $\mathrm{C}_{3} \mathrm{H}$ mice.

\section{$\mathrm{C}_{3} \mathrm{H}$ mice}

Pathogen free, 14-day-old, male $\mathrm{C}_{3} \mathrm{H}$ mice were provided by the Animal Care Laboratory of the Institute of Zoology,

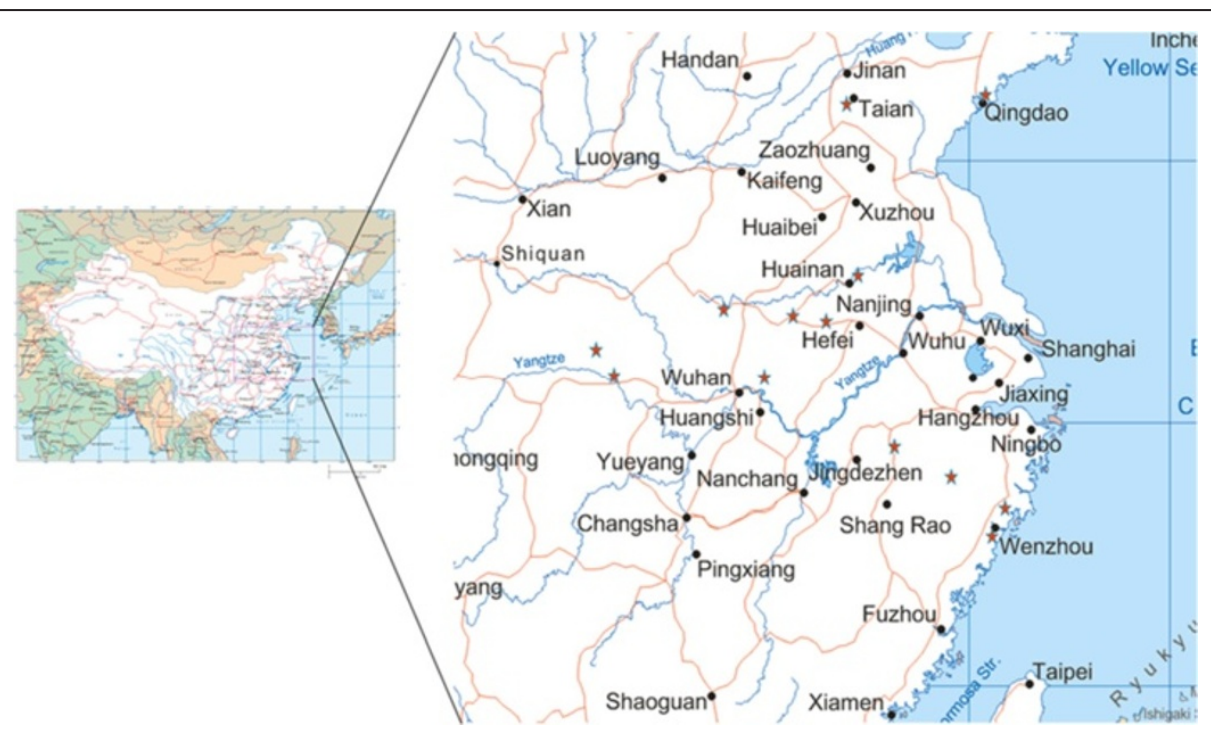

Figure 1 Origins of ticks in the study. Ticks collected from January, 2009 to January 2013 in sites marked with stars on the map. 
Chinese Academy of Science and served as hosts for both $I$. sinensis and $R$. monacensis. $\mathrm{C}_{3} \mathrm{H}$ mice were maintained in accordance with the Institutional Animal Care and Use Committee of Beijing Institute of Microbiology and Epidemiology.

\section{Cultivation $R$. monacensis from ticks}

Hemolymph from ticks was cultured in human embryonic lung (HEL) fibroblasts with the centrifugation-shell vial technique using $12-\mathrm{mm}$ round cover slips seeded with $1 \mathrm{ml}$ of medium containing 50,000 cells and incubated in a $5 \% \mathrm{CO}_{2}$ incubator at $37^{\circ} \mathrm{C}$ for 3 days to obtain a confluent monolayer [20]. Cultures were monitored for 4 weeks, and bacterial growth was assessed every 7 days on cover slips directly inside the shell vial using Gimenez and immunofluorescence staining methods. For positive cultures, the Rickettsia isolate was identified using PCR and sequencing as described below.

\section{Infection I. sinensis with CTF and immersion methods}

Rickettsa-free $I$. sinensis were fixed on slides individually using double-sided adhesive tape. A drawn-our capillary tube filled with $10 \mu \mathrm{L}$ of medium containing 500 cells infected with $R$. monacensis was placed over hypostomes of tick and the tick was allowed to feed at $34^{\circ} \mathrm{C}$ as described for artificial infection by Borrelia burgdorferi [21]. Pipettes were replaced every $2-3 \mathrm{~h}$ for $6 \mathrm{~h}$ and then ticks were detached from the double-sided tape and returned to colony maintenance conditions. A similar procedure was performed to infect the Rickettsia free colony of nymphal I. sinensis. To infect larvae by immersion, $50 \mu \mathrm{L}$ of a medium containing 2,500 cells infected with $R$. monacensis was cracked with an ultrasonic cell disruptor (800 watt, $2 \mathrm{~h}$ ) prior to the immersion procedures. Ticks were placed in this medium and vortexed at medium speed and incubated at $34^{\circ} \mathrm{C}$ for $30 \mathrm{~min}$. To avoid larval flotation the centrifugation was pulsed. Centrifuged larvae were surface disinfected by immersion in a $0.1 \%$ bleach solution for $2 \mathrm{~min}$, washed in distilled $\mathrm{H}_{2} \mathrm{O}$, and returned to colony maintenance conditions [22].

\section{Transovaries Transmission, TOT}

After infection by CTF, every $4 \mathrm{I}$. sinensis females were allowed to feed on one naïve $\mathrm{C}_{3} \mathrm{H}$ mouse along with 4 males. To avoid grooming, each mouse was restrained with a collar. The parasitized mice were reared individually in a cage over water pans, where well fed females were recovered and returned to colony maintenance conditions after dropping from their hosts. The engorged females were maintained individually until egg laying. From each maternal individual, we sampled $300 \mathrm{~F}_{1}$ eggs and allocated them randomly into 3 pools. The rest of the eggs were kept in colony maintenance conditions to hatch. After hatching, $300 \mathrm{~F}_{1}$ larvae were sampled as before and allocated to 3 pools. The filial eggs and resultant larvae pools were submitted to be screened for $R$. monacensis infection with the PCR and sequencing method described below.

\section{Transstadial Transmission, TS}

After infection by CTF or immersion methods, 10 nymphs or 50 larvae of $I$. sinensis were allowed to feed on one $\mathrm{C}_{3} \mathrm{H}$ mouse as described above. A total of 15 $\mathrm{C}_{3} \mathrm{H}$ mice were used as hosts for nymphs and 6 mice were used for larvae. After detachment, the engorged nymphs and larvae were harvested. A total of 30 engorged nymph or 30 engorged larvae were sampled and the rest were maintained individually prior to molting into adult or nymphs. Then, the subsequent 25 males and 25 females or 50 nymphs were also sampled and the rest were used in the following experiment to study transmission from tick to host reservoir. The sampled ticks were tested for $R$. monacensis infection with PCR and sequencing methods as described below.

\section{Transmission from tick to host reservoir}

The transmission competence of tick to naïve mice was tested as follows. Females, nymphs and larvae of I. sinensis obtained from TS and TOT experiments were also allowed to feed on naïve $\mathrm{C}_{3} \mathrm{H}$ mice respectively as described above. At 5 days following tick detachment, blood was collected by tail vein from each mouse to evaluate for $R$. monacensis infection using PCR and sequencing methods.

\section{PCR detection for Rickettsia monacensis in cells, tick and mice and sequencing}

The QIAamp DNA mini Kits (QIAGEN, Hilden, Germany) were utilized to prepare DNA templates from the cells, ticks and mice samples according to the manufacturer's protocol. All PCR assays used Taq polymerase (Promega) in $50 \mu \mathrm{L}$ reactions with the manufacturer's suggested buffer and nucleotide concentrations. Presence of rickettsial DNA in tick and mice blood extracts was detected with specific primers as follows: Primer GltA.877p and GltA1258n [23], which amplify a 382 bp part of gltA gene; Primer Rr17.61 and Rr17.492 [24], which amplify a $438 \mathrm{bp}$ fragmentof $17 \mathrm{kD}$ protein gene; Primer Rr70p and Rr602nfor the 530 bp fragment of the ompA gene; RrompBf and RrompBr for a 515 bp fragment of ompA [23]. The PCR were performed as described previously [23] with distilled water instead of DNA template used as a negative control. All amplicons were cloned into the pGEM-T Easy vector and subjected to bidirectional sequencing (Sangon Biotech, Shanghai, China) with SP6 and T7 promoter primers. The newly obtained sequences were aligned with corresponding sequences retrieved from the GenBank database (http://www.ncbi.nlm.nih.gov) using BioEditv.7.0.5.3. The phylogenetic trees for the genes were 
constructed applying the Neighbour-Joining (NJ) algorithm implemented in the software package MEGA 5.20.

\section{Ethics statement}

The study had received the specific approval of the Institutional Animal Care and Use Committee (IACUC) of Beijing institute of Microbiology and Epidemiology. It was informed of the objectives, requirements and procedures of the experiments. Before each feeding process, a single dose of a non-steroidal anti-inflammatory agent (NSAID) Aspirin was orally administrated to mice to alleviate the suffering of the mice, following the guidance of IACUC of Beijing institute of Microbiology and Epidemiology.

\section{Results}

R. moncacensis prevalence in ticks and its cultivation from I. sinensis hemolymph

A total of 1503 ticks were classified into 5 species belonging to 4 genera. These included $586 \mathrm{I}$. sinensis

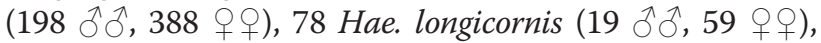

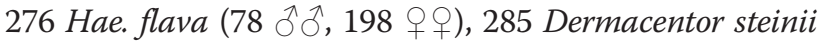

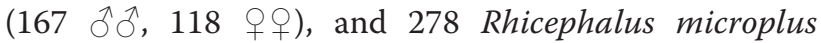
(80 ठํ, 198 우우) (Figure 1). Samples positive for corresponding fragments of both gltA and ompA of SFG Rickettsia spp. were considered SFG rickettsia infections. Using this criterion, the expected DNA fragments of $R$. monacensis were detected in 9 of $586 I$. sinensis ticks, $2 \hat{O}^{\lambda} \delta^{\lambda}(1.06 \%)$ and 7 우 (1.80\%). The difference between sexes was not significant $\left(X^{2}=0.894, P>0.05\right)$. No Hae. longicornis, Hae. flava, Rh. microplus or D. steinii was positive for Rickettsia spp. Similar to the PCR results, cultivation of tick hemolymph to detect $R$. monacensis was only positive for $I$. sinensis, as shown in HEL fibroblasts stained with Gimenez and indirect immunoflourescence assay results (Figure 2). To confirm the positive results of the culture, we sampled cells from the cover slips and used primers targeting gltA gene, ompA gene, ompB gene and $17-\mathrm{kD}$ protein gene respectively and got positive results as expected. All sequences obtained (KF000308 for gltA, KF395227 for ompA, KF800897 for ompB and KF800896 for $17-\mathrm{kDa}$ protein gene) were aligned with known sequences using the BLAST (http://blast.ncbi.nlm.nih.gov/ Blast.cgi) program. The best matches (highest identities) occurred with the corresponding sequence coding genes of $R$. monacensis. Nucleotide sequence identities ranged from 99.93\% to $99.92 \%$ for the $\mathrm{glt}$ A gene, from $99.09 \%$ to $100 \%$ for the ompA gene, from 99.46 to $100 \%$ for the $o m p B$ gene, and from $99.72 \%$ to $100 \%$ for the $17-\mathrm{kDa}$ protein gene, indicating that both the homology levels of the sequencesare within species thresholds for $R$. monacensis proposed by Fournier and others [25]. Sequences related to corresponding reference sequences of the universally recognized SFG group Rickettsia spp. in Genbank were utilized to construct phylogeny trees, the new sequences were clustered into a separate $R$. monacensis branch (Figure 3).

\section{Transmission of $R$. monacensisbyl. sinensis}

The potential pathogenicity of $R$. monacensis to I. sinensis was evaluated by comparison with a control group, fed with the same volume of PBS solutions through capillary tubes. Over $30 \mathrm{~d}$ post CTF, ticks died at approximately the same rate with no significant differences between groups. At $5 \mathrm{~d}$ after tick detachment from mice, all hemolymph samples collected from 20 engorged females of I. sinensis and blood samples from the infested

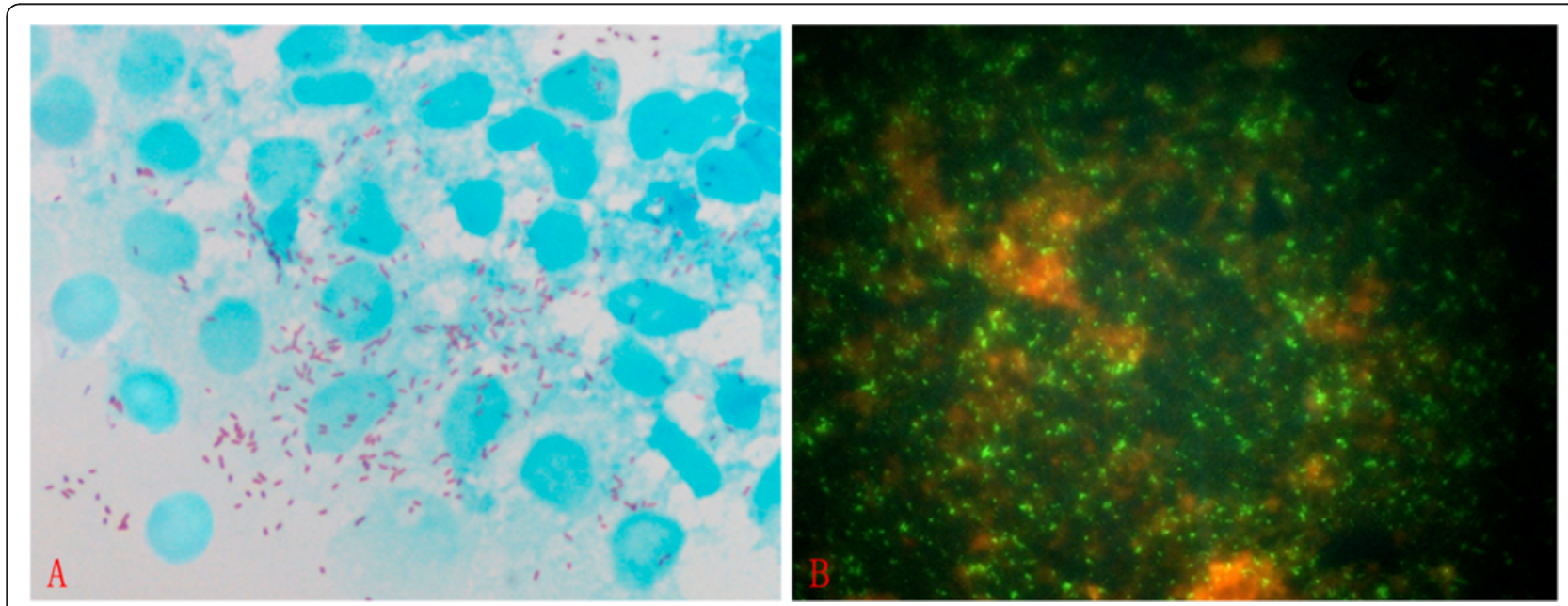

Figure 2 Rickettsia monacensiscultured from I. sinensis. Rickettsia monacensis cultured in HEL fibroblast from the hemolymph of I. sinensis Guangshan, Henan. (Panel A. cell smear stained with Gimenez and Panel B. Indirectly Immunofluorescence Assay with commercial antibody of R. monacensis produced by Vircell, Spain). 


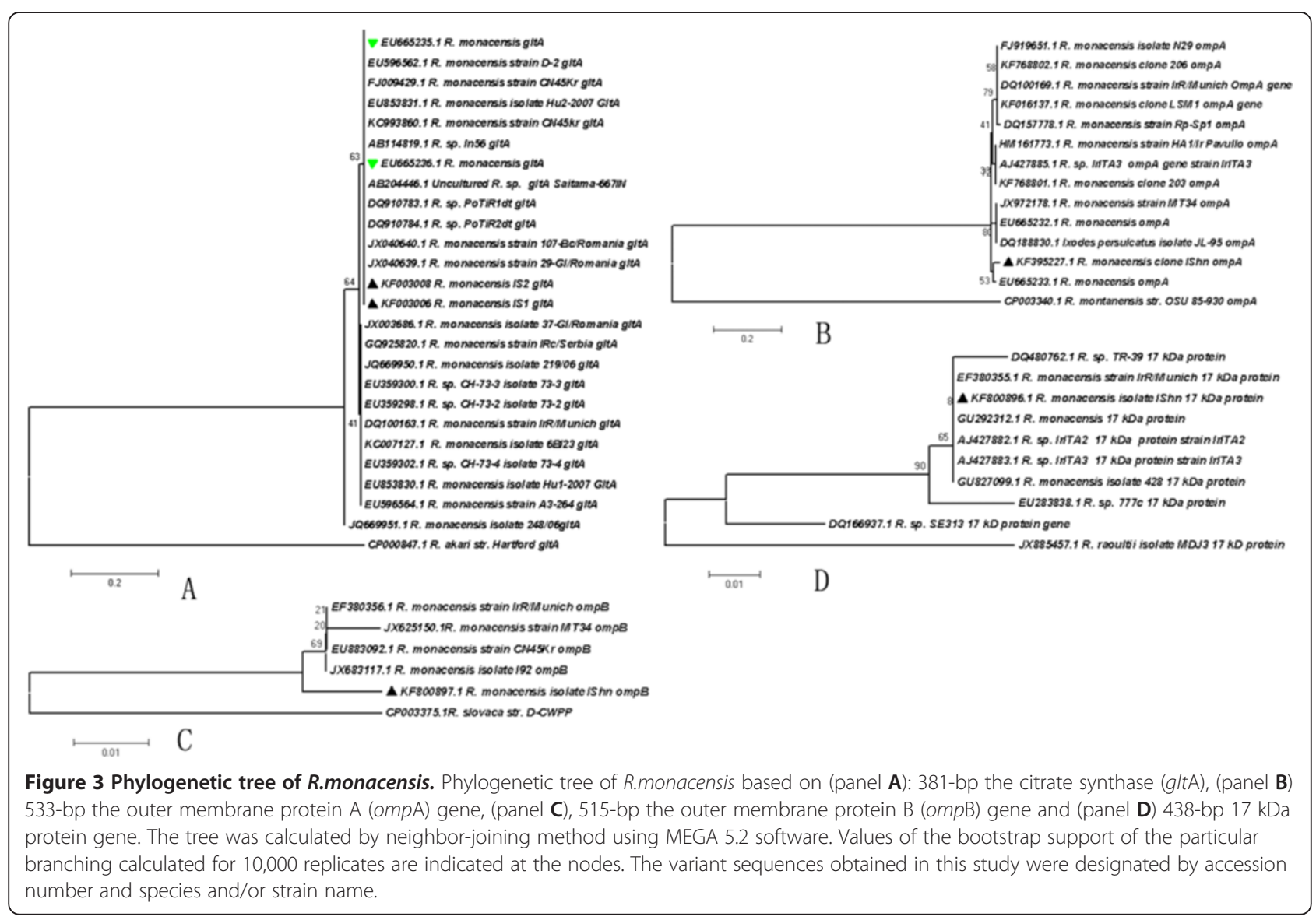

mice were infected with $R$. monacensis, indicating successful infection by CTF. The replete females were maintained individually until egg production at approximately $21 \mathrm{~d}$. All 15 egg pools from 5 females yielded positive results as did the 15 larval ones, suggesting a 100\% TOT infection rate.

We tested for R. monacensis infection in I. sinensis larvae by TS to nymphs by immersing 300 Rickettsia free I. sinensis larvae in medium containing $R$. monacensis. At $7 \mathrm{~d}$ post immersion, a total of 284 larvae survived and there was no significant difference with the control group treated with a PBS solution. After infesting the
$\mathrm{C}_{3} \mathrm{H}$ mice, 186 engorged larvae were harvested. All the 30 sampled engorged larvae were infected with $R$. monacensis. Four weeks later, 145 nymphs were recovered and 29 of 50 sampled nymphs contained Rickettsia. This is a $58.0 \%$ TS infection rate from larval to nymphal stage (Table 1). We fed 160 Rickettsia free I. sinensis nymphs with the medium containing $R$. monacensis by CTF to study transmission from nymphs to adults. At $7 \mathrm{~d}$ post CTF, 148 nymphs survived and these were not significantly different from the control groups. After feeding on the $\mathrm{C}_{3} \mathrm{H}$ mice, 130 engorged nymphs were harvested.

Table 1 Results for transmission experiments

\begin{tabular}{|c|c|c|c|c|c|c|c|}
\hline \multirow{3}{*}{$\begin{array}{l}\text { Stage } \\
\text { From adult to larva }\end{array}$} & \multirow{3}{*}{$\begin{array}{l}\text { Group } \\
\text { Infected group }^{\mathrm{A}}\end{array}$} & \multirow{3}{*}{$\begin{array}{l}\begin{array}{l}\text { No. survived/ } \\
\text { treated }\end{array} \\
21 / 30\end{array}$} & \multirow{3}{*}{$\begin{array}{l}\text { No. engorged } \\
20\end{array}$} & \multicolumn{4}{|c|}{ No. infected/sampled } \\
\hline & & & & \multirow{2}{*}{$\begin{array}{l}\text { Engorged } \\
12 / 12\end{array}$} & \multicolumn{2}{|c|}{ Subsequent stage or sex } & \multirow{2}{*}{$\begin{array}{l}\text { Mice infestec } \\
5 / 5\end{array}$} \\
\hline & & & & & Egg $15 / 15^{*}$ & Larvae $15 / 15^{*}$ & \\
\hline & Control $^{\mathrm{A}}$ & $21 / 30$ & 20 & $0 / 11$ & $\mathrm{Egg} 0 / 15^{*}$ & Larvae0/15* & $0 / 5$ \\
\hline \multirow[t]{2}{*}{ From larva to nymph } & Infected group ${ }^{B}$ & $300 / 300$ & 284 & $30 / 30$ & Nymph 29/50 & & $6 / 6$ \\
\hline & Control $^{B}$ & $300 / 300$ & 282 & $0 / 30$ & Nymph 0/50 & & $0 / 6$ \\
\hline \multirow[t]{2}{*}{ From nymph to adult } & Infected group ${ }^{c}$ & $148 / 160$ & 130 & $28 / 30$ & Male $10 / 25$ & Female 18/25 & $6 / 6$ \\
\hline & Control $^{C}$ & $145 / 160$ & 129 & $0 / 30$ & Male $0 / 25$ & Female0/25 & $0 / 6$ \\
\hline
\end{tabular}

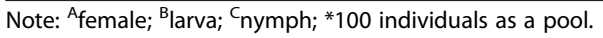


A sample of 30 engorged nymphs was all infected with R. monacensis. At $42 \mathrm{~d}, 88$ adults were recovered and 10 of 25 males and 18 of 25 females sampled were positive, indicating a $56.0 \%$ TS infection rate from nymph to adult (Table 1). All 17 mice fed by infected I. sinensis, 5 by larvae, 6 by nymphs and 6 by adults, were Rickettsia positive, as determined by PCR, indicating the $100 \%$ transmitting efficiency from tick to host reservoir (Table 1).

\section{Discussion}

Mediterranean Spotted like Fever and its pathogen $R$. monacensis has been well characterized in previous studies and natural infection of $R$. monacensis in many tick species has also been confirmed in many countries. However, the details of the maintenance and transmission of $R$. monacensis in ticks remain incomplete. In Eurasia, I. ricinus has been regarded as the vector in many MSF like cases. Due to its close morphological and phylogenic relationships with I. ricinus, in America I. scapularis, was considered a vector candidate in assessing the possible transmission mechanism by Baldridge et al. [26]. Using green fluorescent protein (GFP) expressing $R$. monacensis Rmona658, they demonstrated the transmission of $R$. monacensis in I. scapularis from larvae to nymphs and nymphs to adults. However, TOT and horizontal transmission failed. Rmona658 did not establish in small mammal hosts by the feeding of either I. scapularis nymphs or adults. Thus no other tick species except $I$. ricinus has been demonstrated to be a vector of $R$. monacensis until the present report.

Our results estimate the natural infection of $I$. sinensis with $R$. monacensis in Chinese locations, suggesting that MSF like rickettsiosis occurs in both central and southern China. The potential threat of $R$. monacensis should be considered in differential diagnosis in spotted fever patients. Considering natural infection and experimental transmission evidence from TS, TOT and horizontal transmission protocols, I. sinensis appears to be a competent vector. I. sinensis has frequently been recorded feeding on residents and tourists in central and southern China $[27,28]$ so the potential health risks of $I$. sinensis and $R$. monacensis should be recognized by public health authorities. The vector competence of I. sinensis helps to explain the trend of southward expanding range of MSFlike rickettsiosis in China. The valid geographic range of I. sinensis is not limited to the areas sampled in this report [27,29], therefore, MSF-like rickettsiosis might occupy larger geographical areas in China.

In our survey, R. monacensis was only found in I. sinensis; but it has previously been recorded in I. persulcatus [14]. No I. persulcatus were collected in the present study and this species mainly occurs in the north and northeast China [27]. The southern range limit of I. persulcatus is in the Qiling-Taihang-Yanshan Mountains [27,28].
Our survey sites did not include this range and we collected neither $R$. sibrica nor $R$. heilongjiangensis, which often co-occurs with $I$. persulcatus. Thus, we assume misidentification of ticks might have occurred in the report of Li et al. [14] because of the morphological similarity of the two species (they belong to the same complex) and despite the fact that temporal and spatial factors and even the low prevalence might contribute to the differences.

I. sinensis belongs to the I. ricinus species complex which is important in the animal to human transmissionof tick borne pathogens such as, Borrelia burgdorferi, Babesia protozoans, Anaplasma phagocytophilum. I. sinensisis the principal vector of the Lyme disease agent Borrelia burgdorferi and related Borrelia species in southern China [27]. Because the transmission cycle of $R$. monacensis by I. sinensis appears similar to that of Borrelia spp, co- infection of humans by $R$. monacensis and other tick borne pathogens might occur in some regions. The co-infection prevalence in human populations and the related public health risks will require further investigation.

\section{Conclusions}

As demonstrated by natural infection and transmission studies, $I$. sinensis is a competence vector for $R$. monacensis, the agent for Mediterranean Spotted Fever like rickettsiosis.

\section{Competing interests}

The authors declare that they have no competing interests.

\section{Authors' contributions}

YS carried out the molecular genetic studies, participated in the sequence alignment and drafted the manuscript. XW participated in the sequence alignment. WCC participated in the design of the study and performed the statistical analysis. XDY and WDJ conceived of the study, and participated in its design and coordination and helped to draft the manuscript. All authors read and approved the final manuscript.

\section{Acknowledgements}

We are grateful to Prof. G Xu for reviewing the manuscript. We thank LetPub for its linguistic assistance during the preparation of this manuscript. This study was supported by the National Science Foundation of China (30400364, 81271878), Special Fund of the Ministry of Health of P. R. China (Grant no. 201202019) and National Critical Project for Science and Technology on Infectious Disease of P. R. China (Grant No.2012ZX10004219) for funding the research.

\section{Author details}

${ }^{1}$ State Key Laboratory of Pathogen and Biosecurity, Beijing Institute of Microbiology \& Epidemiology, No. 20 Dongdajie Str. Fengtai District, Beijing, People Republic of China. ${ }^{2}$ Centre for Disease Control and Prevention of Jindong, No.295 Jiangjun Road, Jinhua, Zhejiang province, People Republic of China. ${ }^{3}$ Center for Health Inspection, Heilongjiang Bureau of Entry \& Exit Inspection and Quarantine, No.9 Ganshui Road, Harbin, People Republic of China. ${ }^{4}$ Center for Disease Control and Prevention of Wenzhou, No. 59 Yingdaoguan Rd., Lucheng District, Wenzhou, Zhejiang province, People Republic of China.

Received: 11 June 2014 Accepted: 30 October 2014

Published online: 19 November 2014 
References

1. MerhejVand Raoult D: Rickettsial evolution in the light of comparative genomics. Rev Camb Philos Soc 2011, 86:379-405.

2. Raoult D, Parola P: Rickettsial Diseases. New York: Informa Healthcare USA Inc; 2007.

3. Ricketts HT: The transmission of Rocky Mountain spotted fever by the bite of the wood tick (Dermacentor occidentalis). J Am Med Assoc 1906 47:358.

4. Simser JA, Palmer AT, Fingerle V, Wilske B, Kurtti TJ, Munderloh UG: Rickettsia monacensis sp. nov., a Spotted Fever Group Rickettsia, from Ticks (Ixodes ricinus) Collected in a European City Park. Appl Environ Microbiol 2002, 68:559-4566.

5. Jado I, José $A O$, Mikel A, Horacio G, Raquel E, Valvanera I, Joseba P, Aranzazu P, María JL, Cristina GA, Isabel RM, Pedro A: Rickettsia monacensis and Human Disease, Spain. Emerg Infect Dis 2007, 13:1405-1407.

6. Madeddu G, Fabiola M, Antonello C, Alessandra C, Sergio B, Ivana M, Maria LF, Giovanni R, Maria SM: Rickettsia monacensis as cause of Mediterranean spotted fever-like illness. Italy Emerg Infect Dis 2012, 18:702-704.

7. Tijsse-Klasen E, Hein S, Nenad P: Co-infection of Borrelia burgdorferi sensu lato and Rickettsia species in ticks and in an erythema migrant's patient. Parasit Vectors 2013, 6:347.

8. Márquez FJ: Spotted fever group Rickettsia in ticks from southeastern Spain natural parks. Exp Appl Acarol 2008, 45:185-194.

9. Beninati L, Sacchi L, Genchi C, Bandi C: Emerging rickettsioses. Parassitologia 2004, 46:123-126. Article in Italian.

10. Dobler G, Essbauer S, Wolfel R: Isolation and preliminary characterization of 'Rickettsia monacensis' in south-eastern Germany. Clin Microbiol Infect Dis 2009, 15:263-264.

11. Rymaszewska A, Piotrowski M: Use of DNA sequences for Rickettsia identification in Ixodes ricinus ticks: the first detection of Rickettsia monacensis in Poland. Microb Infect 2013, 15:140-146.

12. Christova I, Pol J, Van D, Yazar S, Velo E, Schouls L: Identification of Borrelia burgdorferi sensu lato, Anaplasma and Ehrlichia species, and spotted fever group Rickettsiae in ticks from Southeastern Europe. Eur J Clin Microbiol Infect Dis 2003, 22:535-542.

13. Sekeyova Z, Fournier PE, Rehacek J, Raoult D: Characterization of a new spotted fever group rickettsia detected in Ixodes ricinus (Acari: Ixodidae) collected in Slovakia. J Med Entomol 2000, 37:707-713.

14. Li W, Liu L, Jiang X, GuoX GM, RaoultD PP: Molecular identification of spotted fever group Rickettsiae in ticks collected in central China. Clin Microbiol Infect 2009, 15:279-280.

15. Shin SH, Seo HJ, Choi YJ, Choi MK, Kim HC, Klein TA, Chong ST, Richards AL, Park KH, Jang WJ: Detection of Rickettsia monacensis from Ixodes nipponensis collected from rodents in Gyeonggi and Gangwon Provinces, Republic of Korea. Exp Appl Acarol 2013, 61:337-347.

16. Lee KM, Choi YJ, Shin SH, Choi MK, Song HJ, Kim HC, Klein TA, Richards AL, Park KH, Jang WJ: Spotted fever group rickettsia closely related to Rickettsia monacensis isolated from ticks in South Jeolla province, Korea. Microbiol Immunol 2013, 57:487-495.

17. Jia N, Zheng $Y C$, Ma L, Huo QB, Ni XB, Jiang BG, Chu YL, Jiang RR, Jiang JF, Cao WC: Human Infections with Rickettsia raoultii, China. Emerg Infect Dis 2014, 20:866-868.

18. Patton TG, Dietrich G, Brandt K, Dolan MC, Piesman J, Gilmore RD: Saliva, salivary gland, and hemolymph collection from ixodes scapularis ticks. J Vis Exp 2012, 60:e3894.

19. Kurtti TJ, Munderloh UG, Hughes CAN, Engstrom SM, Johnson RC: Resistance to tick-borne spirochete challenge induced by Borrelia burgdorferi strains that differ in expression of outer surface proteins. Infect Immun 1996, 64:4148-4153.

20. da Quesa M, Sanfeliu I, Cardeñosa N, Segura F: Ten years' experience of isolation of Rickettsia spp. from blood samples using the shell-vial cell culture assay. Ann N Y Acad Sci 2006, 1078:578-581.

21. Korshus JB, Munderloh UG, Bey RG, Kurtti TJ: Experimental infection of dogs with Borrelia burgdorferi sensu stricto using Ixodes scapularis ticks artificially infected by capillary feeding. Med Microbiol Immunol 2004 193:27-34.

22. Mitzel DN, Wolfinbarger JB, Daniel Long R, Max M, Best SM, Bloom ME: Tick-borne flavivirus infection in Ixodes scapularis larvae: development of a novel method for synchronous viral infection of ticks. Virology 2007, 365:410-418.
23. Regnery RL, Spruill CL, Plikaytis BD: Genotypic identification of rickettsiae and estimation of intraspecies sequence divergence for portions of two rickettsial genes. J Bacteriol 1991, 173:1576-1589.

24. Noda H, Munderlonh UG, Kurtti IJ: Endosymbionts of ticks and their relationship to Wolbachia spp and tick-borne pathogens of human and animals. Appl Environ Microbiol 1997, 63:3926-3932.

25. Fournier PE, Dumler JS, Greub G, Zhang JZ, Wu YM, Raoult D: Gene Sequence-Based Criteria for Identification of New Rickettsia Isolates and Description of Rickettsia heilongjiangensis sp. nov. J Clin Microbiol 2003, 41:5456-5465.

26. Baldridge GD, Kurtti TJ, Burkhardt N, Baldridge AS, Nelson CM, Oliva AS, Munderloh UG: Infection of Ixodes scapularis ticks with Rickettsia monacensis expressing green fluorescent protein: a model system. J Invertebr Pathol 2007, 94:163-174.

27. Teng KF, Jiang ZJ: Economic insect faunaof China, Acari, Ixodidae. In Ixodidae. Edited by Teng IKF, Jiang ZJ. Beijing: Science Press; 1991:81-234

28. Sun Y, Xu RM, Cao WC: Ixodes sinensis: competence as a vector to transmit the Lyme disease spirochete Borrelia garinii. Vect Born Zoon Dis 2003, 3:39-44.

29. Guglielmone AA, Robbins RG, Apanaskevich DA, Petney TN, Estrada-Peña A, Horak IG: The Hard Ticks of the World (Acari: Ixodida: Ixodidae). Dordrecht, Netherlands: Springer Science; 2014

doi:10.1186/s13071-014-0512-8

Cite this article as: Ye et al:: Vector competence of the tick Ixodes sinensis (Acari: Ixodidae) for Rickettsia monacensis. Parasites \& Vectors 2014 7:512

\section{Submit your next manuscript to BioMed Central and take full advantage of:}

- Convenient online submission

- Thorough peer review

- No space constraints or color figure charges

- Immediate publication on acceptance

- Inclusion in PubMed, CAS, Scopus and Google Scholar

- Research which is freely available for redistribution 